case report

\title{
Gastric gastrointestinal stromal tumour
}

\section{Ivan Žokalj ${ }^{1}$, Renata Čulinović-Čaić ${ }^{2}$, Zvonimir Magaš ${ }^{2}$, Zlatko Pavčec ${ }^{1}$, Hussein Saghir ${ }^{1}$, Jasminka Igrec ${ }^{1}$, Miljenko Marotti ${ }^{3}$}

\author{
${ }^{1}$ Department of Radiology and Ultrasound, County Hospital Čakovec, Croatia; \\ ${ }^{2}$ Department of Surgery, County Hospital Čakovec, Croatia \\ ${ }^{3}$ Department of diagnostic and interventional radiology, \\ University Hospital "Sisters of Mercy", Zagreb, Croatia
}

\begin{abstract}
Background. Gastrointestinal stromal tumours (GISTs) are the most common mesenchymal tumours of the digestive tract. These tumours can not be simply divided into benign and malignant forms. There is a continuum from benign to malignant forms. The tumour size and mitotic activity have strong influence on GISTs behaviour. Tumour behaviour also varies according to the site of the origin. The site of the tumour origin can be anywhere along the digestive tract, in the mesentery or omentum. GISTs are mainly benign tumours, about 70-80\%, usually found in the gastric wall. We report a case of 70-year old female with gastric form of GIST.

Case report. The patient has undergone abdominal ultrasound (US) because of a palpable lump in the epigastrium, which mainly revealed hyperechogenic round mass with small hypoechogenic areas in the central part. On the abdominal computed tomography (CT) a large expansive mass with heterogeneous structure was depicted in the gastric wall. The mass had higher attenuation coefficients on the periphery and lower in the central part. During the surgery the large exophytic tumour of the gastric wall has been found. The diagnosis of gastric GIST has been obtained after the patohistologic and imunohistochemical analysis.

Conclusions. Gastrointestinal stromal tumours may be the statistically rare tumours $(0.1 \%-0.3 \%$ of all gastrointestinal tumours) but when we have the patient with a round, mainly exophytic mass on the wall of the gastrointestinal tract or peritoneum, GIST must be taken into consideration. Cross-sectional imaging methods like US and CT allow the preoperative diagnosis of the tumour and staging.
\end{abstract}

Key words: gastrointestinal stromal tumours; computed tomography

\section{Introduction}

Received 3 November 2008

Accepted 18 November 2008

Correspondence to: Ivan Žokalj, MD, Department of Radiology and Ultrasound, County Hospital Čakovec, Ivana Gorana Kovačića 1e, 40000 Čakovec, Croatia. Phone: +385 40375 297; E-mail: ivan.zokalj@ck.t-com.hr
Gastrointestinal stromal tumours (GISTs) are currently defined as mesenchymal tumours of the gastrointestinal tract, mainly KIT (CD117)-positive. ${ }^{1}$ These tumours usually affect the population over 50 years, rarely patients younger than 40 years of age, and are extremely rare in the child- 
hood. ${ }^{1-3}$ The frequency of GISTs is about 10 to 20 cases per million persons, according to the Miettinen and Lasota. ${ }^{4}$ GISTs can arise anywhere through the gastrointestinal tract but they can also occur in the omentum, mesentery and retroperitoneum as a primary tumour. In $70 \%$ of cases GISTs arise in stomach, the second most frequent site is small intestine (20-30\%). Other less frequent sites are anus, rectum, colon and oesophagus. ${ }^{4}$ GISTs of gastrointestinal tract commonly arise in the muscularis propria of the stomach or intestinal wall, usually in the outer muscular layer. That is the reason why GISTs have an exophytic growth pattern in majority of the cases and usually manifest themselves as large extraluminal abdominal masses. Clinical features of GISTs depend on the size and anatomic location of the tumour. They can be presented with mild signs and symptoms of anaemia caused by occult bleeding in the gastrointestinal tract or they can have a dramatic picture with hematemesis, melena, abdominal pain and signs of intestinal obstruction. ${ }^{5,6}$

Histogenetically gastrointestinal stromal tumours probably originate from interstitial cells of Cajall (or the native KIT-positive gut pacemaker cell) which intermediates between the GI autonomic nervous system and smooth muscle cells regulating GI motility and autonomic nerve function. This postulate is made on the expression of KIT on GISTs tumour cells., ${ }^{1,7}$

Larger forms of GISTs are usually centrally cystic, positioned extraluminally, while smaller GISTs are subserosal and intramural solid tumours, rarely polypod intraluminal ones. ${ }^{1}$ GISTs can generally be divided into three groups: spindle cell type (70\%), epithelioid type (20\%) and mixed spindle and epithelioid cel type. ${ }^{7}$ Gastric GISTs can be histologically divided into two groups, four spindle cell subtypes (sclerosing spindle cell, palisaded-vacuolate spindle cell, hypercellular spindle cell, sarcomatous spindle cell) and four epitheloid subtypes (sclerosing epithelioid GIST with syncytitial pattern, epitheloid GIST with dyscohesive pattern, hypercellular epitheloid GIST, sarcomatous epitheloid GIST). Sarcomatous subtype is highly cellular form of GIST with a marked mitotic activity (more than 20 per 50 HPFs). ${ }^{9}$ The most important feature for immunohistochemical differentiation between GISTs and other similar soft-tissue tumours are the antigens on the surface of the tumour cells, especially KIT tyrosine kinase receptor (CD117) which has been found in more than 95\% of GISTs. There are other antigens which are less specific for GISTs but are commonly expressed, like CD34 and nestin. GISTs sometimes express smooth muscle cell markers (like smooth muscle cell actin-SMA), but are usually negative for desmin, the muscle type intermediate filament protein. ${ }^{1}$ KIT imunoreactivity has shown to be very useful in the treatment of GISTs with immunotherapy as well as in diagnostics. The immunotherapy of GISTs is based on targeting of tyrosine kinase receptors with a selective KIT-tyrosine kinase inhibitor (STI-571, imatinib mesylate). The drug acts as a selective c-kit blocker. ${ }^{6}$

The contrast-enhanced computed tomography (CT) is currently widely available diagnostic imaging method and the imaging modality of choice for patients with abdominal mass suspected for GISTs. ${ }^{7}$

The standard initial treatment for GISTs, even in non-resectable cases, is the surgical treatment. Some authors propose the immediate treatment with imatinib for unresectable and/or metastatic disease. ${ }^{8}$

\section{Case report}

A 70-year old female patient underwent abdominal ultrasound (US) because of pal- 


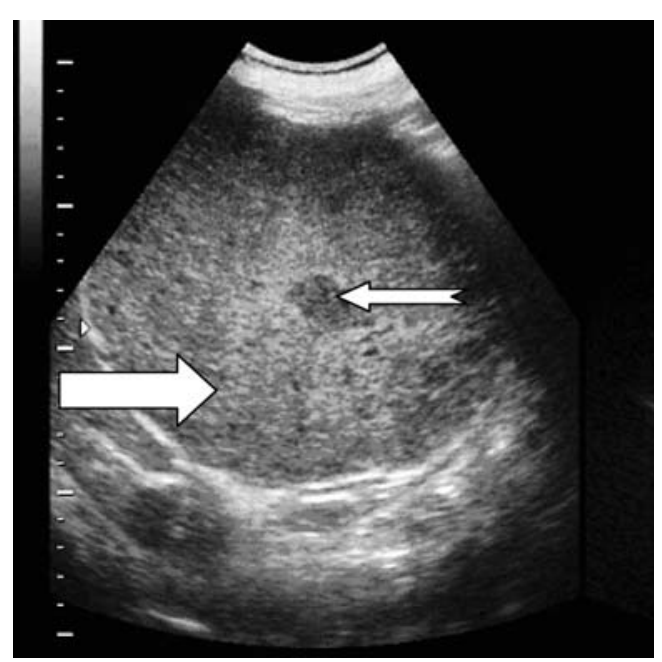

Figure 1. Abdominal ultrasound - axial plain. Large round hyperechogenic expansive mass (white arrow) with small hypoechogenic areas in the central part (white notched arrow).

pable mass in the upper abdomen. The patient suffered from inapetency without vomiting and weight loss, hematemesis or melena. US revealed a large round hyperechogenic expansive mass with small hypoechogenic areas in the central part. There were no signs of focal lesions in parenchymal abdominal organs or lymphadenopathy (Figure 1).

The next step in diagnostics was computed tomography (CT) of the abdomen, performed with three postcontrast phases and distension of gastrointestinal (G-I) tract with $1000 \mathrm{ml}$ of water. CT depicted a large intraperitoneal mass in the upper abdomen, ventrally placed and sharply demarcated from surrounding structures except from stomach. The lesion had a heterogeneous structure on native and postcontrast scans with higher attenuation coefficients on periphery and lower in the central part (about $30 \mathrm{HU}$ on precontrast and $60 \mathrm{HU}$ on postcontrast scans). There were no significant changes in attenuation values centrally between precontrast and all series of postcontrast scanns. No lymphadenopathy or focal lesions in the parenchymal organs have been found. The working (imaging) diagnosis of mesenchymal tumour of the stomach wall (probably GIST) has been established (Figure 2).

The upper G-I tract follow through with barium showed elongated stomach with sharply demarcated contours and concavely impressed lesser curvature (Figure 3).

The patient has been treated surgically with a complete gross resection. During the explorative laparatomy a large tumour in the upper abdomen with origins on the lesser curvature of the stomach without lymphadenopathy has been found (Figure 4).

The material obtained during surgery has been sent to the pathohystologic analysis and immunochemical evaluation. The gross pathologic feature of the specimen was an encapsulated mass of medium firm consistency, measuring $18 \mathrm{~cm}$ in largest diameter. On cut section the tumour was whitish and greyish with dispersed areas of swirling structure. Predominant histological features were spindle shaped cells with scant cytoplasm and big nucleus without nucleolus. The tumour cells formed swirling structures and tracks. Focal areas of degeneration and small areas of tumour tissue necrosis were found.

Three mitoses per 50 consecutive high power fields (HPF) have been detected.

The immunohistochemical analysis showed that the tumour cells were CD117(Ckit), CD 34 and BCL2 positive, SMA -/+ and S-100 negative. The diagnosis of gastrointestinal stromal tumour was established.

\section{Discussion}

GIST is usually placed in the stomach and small intestine. The gastric form is especially common between other possible locations, 50 to $70 \%$ of GISTs are located in the stomach, $33 \%$ of cases in the small 


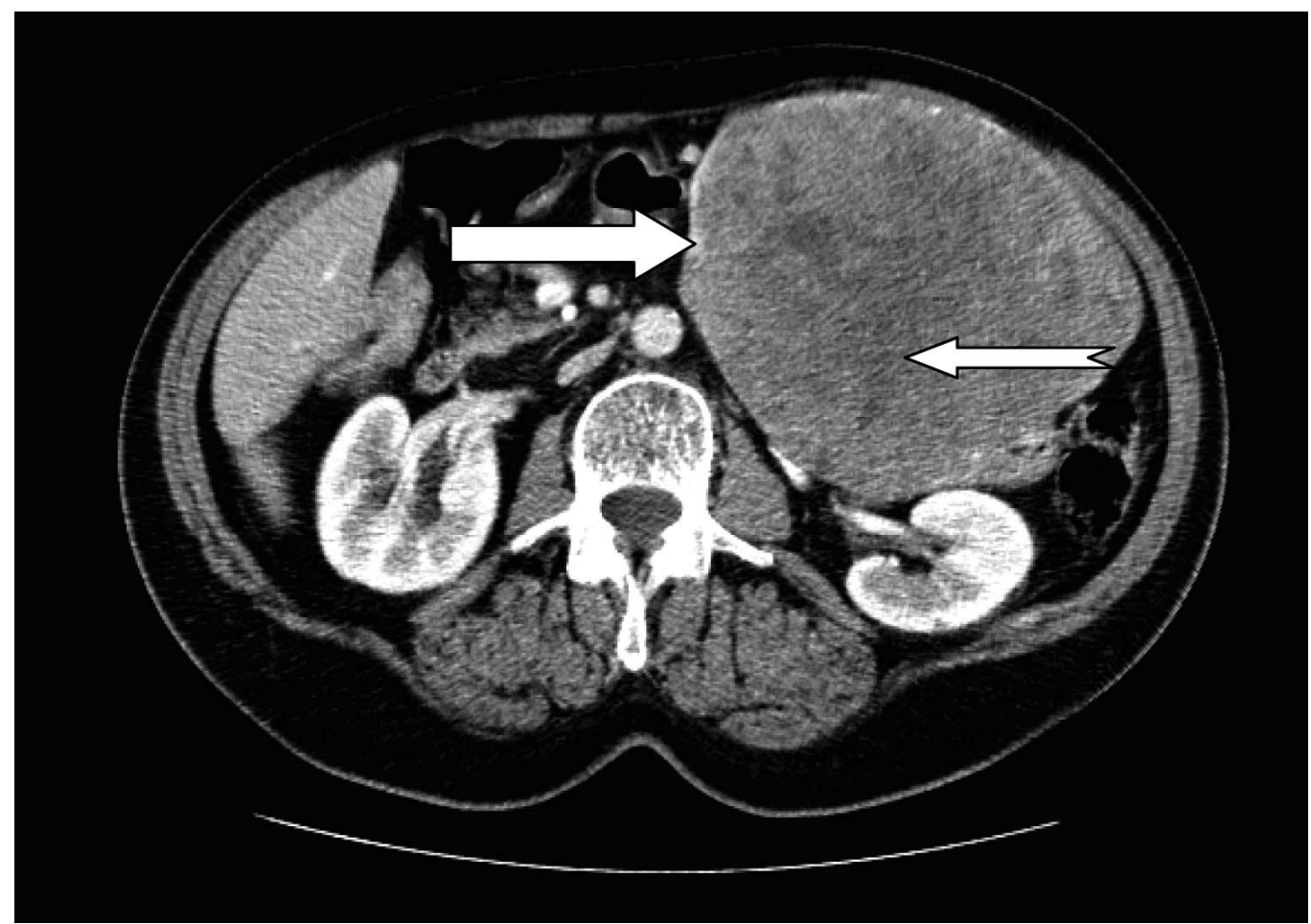

Figure 2. Abdominal CT post-contrast axial scan. Large round expansive mass in the upper abdomen with heterogeneous structure sharply demarcated from surrounding structures depicted on this scan. Higher attenuation coefficients on periphery (white arrow) and lower in the central part were detected on native and postcontrast scans (white notched arrow).

intestine, 5 to $15 \%$ in rectum and colon and only 1 to $5 \%$ in oesophagus. ${ }^{2,10}$ GISTs can originate outside of the gastrointestinal wall, in mesentery or peritoneum in $10 \%$ of cases, but they are extremely rare retroperitoneally. Both sexes are equally affected by GISTs, commonly between 40 and 70 years. The small intestine GISTs are usually more aggressive and have a more grave prognosis than GISTs which originate in other segments of G-I tract. ${ }^{3,11}$

The factors that worsen the prognosis of GISTs are location (all extragastric locations), size of the tumour (bigger than $5 \mathrm{~cm})$, advanced age of the patient, metastasis at the time of tumour manifestation, tumour tissue necrosis and high mitotic index (more than five mitosis per 50 highpower fields). ${ }^{4,10,11}$ In 10 to $30 \%$ of GISTs malignant forms have been diagnosed. ${ }^{4}$ The two most important factors that influence GISTs prognosis are the tumour size and mitotic index. Currently it is believed that only tumours with diameter smaller than $1 \mathrm{~cm}$ can be considered as definitely benign on clinicopathologic features. ${ }^{12}$ The patient presented in this report has few bad prognostic factors, age of 70 years and the tumour largest diameter of $18 \mathrm{~cm}$.

Clinical features of GISTs depend on the tumour size and anatomic location of the tumour, they can be mild signs and symptoms of anaemia caused by occult bleeding in the gastrointestinal tract or dramatic picture with hematemesis, melena, abdominal pain and signs of intestinal obstruction. ${ }^{5}$ GIST of the stomach, small and large intestine and anorectum usually manifest with gas- 
trointestinal bleeding from mucosal ulcerations. The other common clinical features are abdominal pain, nausea, dysphagia, vomiting, weight loss and palpable abdominal mass. ${ }^{13,14}$ The leading clinical feature of the case presented in this article was palpable abdominal mass in the epigastrium. G-I tract obstruction can be manifested in 10 to $30 \%$ of cases, usually in the cases of the small intestine GIST. The biliary tract and the renal obstruction may be the clinical feature of the duodenal GIST. ${ }^{13,15}$

The tumour is usually covered with an affected organ serosa. In the cases of the surrounding structures infiltration by the GIST the primary tumour has more often smooth and broad pushing than insinuating fascicles. The typical location of the first recurrence of GIST is in the abdomen. Liver is the most common metastatic site, in 65\% of cases. The lung and bone metastases usually develop later than liver metastases. Lymph node metastases are very rare for the difference to lymphoma and leiomyosarcoma. ${ }^{1,12,16}$

Computed tomography (CT) and magnetic resonance imaging (MRI) are used for the radiologic diagnostic evaluation of patients with abdominal mass suspected for GIST. Contrast-enhanced CT is currently an imaging method of choice for diagnosing of GISTs, staging and surgical planning because of its wide availability. Endoscopic ultrasound can be used for the evaluation of the local extent in patients with small tumours which have been found incidentally during endoscopy. MRI is a better imaging method for the evaluation of patients with rectal GISTs. MRI is indicated for the evaluation of liver lesions which can not be characterized definitely on CT scans and in cases of contrast-enhance CT is contraindicated. $8,17,18$ The factors that influence CT features of GISTs are size of the tumour, aggressiveness of the tumour and time of presentation during the course of

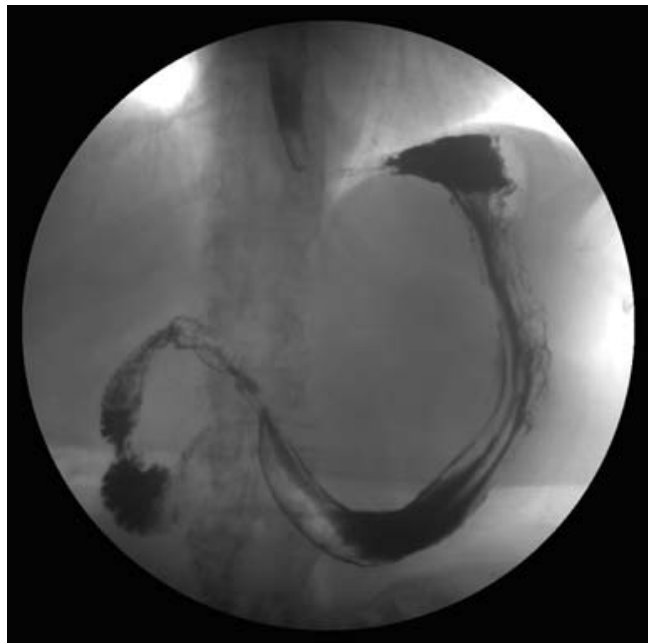

Figure 3. Upper gastrointestinal tract follow through with barium - anteroposterior projection - elongated stomach with sharply demarcated contours and concavely impressed lesser curvature.

the disease. ${ }^{18}$ The typical CT finding of primary GIST is a large mass with heterogeneous structure because of usually centrally placed necrosis, haemorrhage, or cystic degeneration. In fact, the tumour can be so large that it is difficult to define its origin. On postcontrast scans the tumour is usually presented as a hypervascular and wellenhancing heterogeneous mass. Ulceration and fistulisation to the lumen of GI tract are often seen in GIST patients. Small GISTs are commonly presented as homogenous masses. ${ }^{18}$ MRI can be used to follow-up the operated patients and to evaluate response in the patients on an adjuvant therapy with imatinib. Fluorine-18-fluorodeoxyglucose (FDG) positron emission tomography (PET) is recommended in patients on imatinib therapy which need an early detection of tumour response to imatinib because of the possibility for the surgical resection after the cytoreduction with imatinib. ${ }^{17}$ There is no need to perform PET scan in every GIST patient after the complete surgical resection of the tumour. PET can be performed 


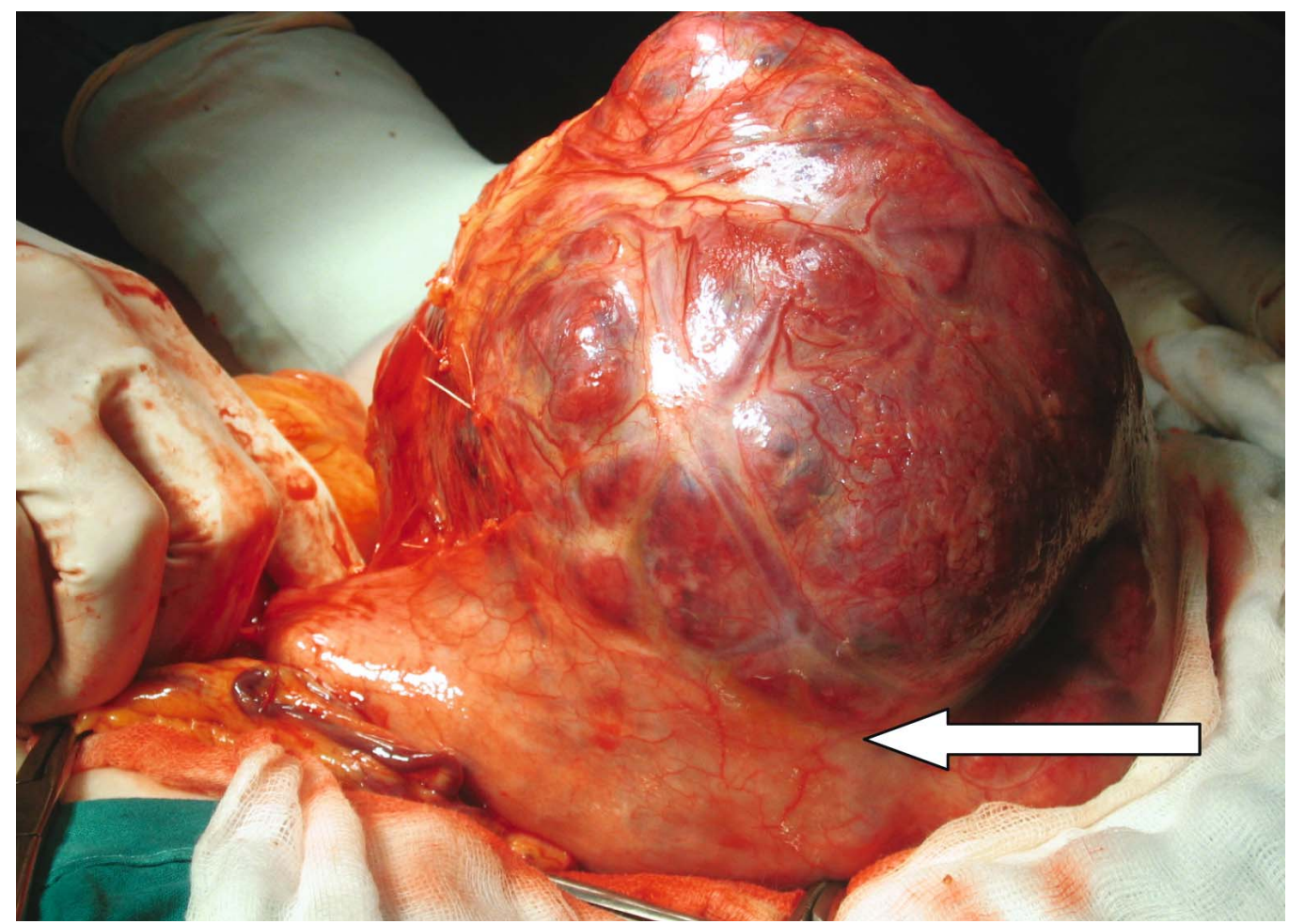

Figure 4. Explorative laparatomy - a large tumour in the upper abdomen with origins on the lesser curvature of the stomach (white arrow).

on patients with CT or /and MRI images suspected for metastases. ${ }^{8,17,18}$

The imaging diagnosis of GIST can be suggested when CT depicts a large, mainly extraluminal tumour arising from the stomach or small bowel wall with heterogeneous contrast material enhancement (with higher attenuation coefficients peripherally and lower coefficients in the central part). Metastases are usually presented in the liver and on the peritoneum, but a lymph node enlargement is very rarely depicted. ${ }^{18,19,20} \mathrm{~A}$ triphasic CT scanning technique with scanning of the native scans and post-contrast scans after the intravenous injection of contrast material in an arterial and a porto-venous phase with delays of 20-30 s and 70$80 \mathrm{~s}$, respectively, is preferred for baseline scans and follow-ups during and after the treatment. ${ }^{17,18}$
Unlike GISTs, lymphomas usually cause lymph node enlargement and circumferential bowel wall thickening which homogenously enhances on post-contrast scans. Carcinoid tumours commonly arise in the terminal ileum often stimulating a desmoplastic reaction with calcifications. A local infiltration and a visceral obstruction are common radiologic features of the carcinoma, very often in large tumours. Metastases of the bowel wall are usually multifocal masses in patients with a history of primary known malignancy. ${ }^{18,20,21}$

It is still difficult to make a differentiation between GIST and other soft-tissue tumours like leiomyosarcoma, intraabdominal fibromatosis of the bowel wall, malignant tumours of nerve sheath and the tumours of vascular origin with radiological and pathological methods. ${ }^{21}$ 
Pathohystology accomplished with immunohystochemistry still remain the gold standard for diagnosing of GIST. The transabdominal biopsy is not recommended because of the possibility of seeding the tumour cells. ${ }^{16}$

GISTs are commonly positive for KIT (CD117), a tyrosine kinase growth factor receptor. KIT positivity is usually strong and pancytoplasmic, but some epithelioid forms of GISTs of the stomach may be weakly CD117 positive or even negative. ${ }^{1}$ We must be aware and analyse the KIT positivity together with other clinical signs, imaging method findings and immunohistochemical test results because there are few other tumours which are generally CD117 positive like small cell carcinoma of the lung, mastocytoma, seminoma and extramedullary myeloid tumour. Tumours like metastatic melanoma, clear cell sarcoma, Ewing sarcoma family of tumours, childhood neuroblastoma and angiosarcoma also sometimes express CD 117. ${ }^{22}$

Among other GIST markers CD34, the hematopoetic progenitor cell antigen plays an important role because it can be found in 80 to $85 \%$ of gastric GISTs and $50 \%$ of small intestinal GISTs. CD34 is usually expressed on endothelial cells, subsets of fibroblasts and neoplasms related to these cell types. ${ }^{9}$ Small intestinal GISTs more often than gastric GISTs express muscle cell markers like SMA. Smooth muscle fibers can be interspersed with SMA and desmin positive GIST intratumoral spindle cells during the infiltration of tumour; in this situation we can have a false muscle marker positivity. The SMA positivity is a favourable prognostic factor for the gastric and small intestinal forms of GIST. The detection of S100 protein is relatively rare in GISTs, it seems to be an adverse prognostic factor in gastric GISTs. ${ }^{1,9}$ Nestin is a type VI intermediate filament protein typical of many stem cells which can be found in most of the cases of GISTs but also in GI schwanomas. ${ }^{23}$

The surgical resection is the standard initial treatment for GISTs. Until the beginning of an application of imatinib (the specific tyrosine kinase inhibitor) in GIST therapy, surgery was the only way of the treatment because the conventional chemotherapy and the radiation therapy proved inefficient. Generally speaking about $85 \%$ of patients with primary localized form of GIST, they can be treated with a complete gross resection, but approximately $50 \%$ of those patients develop the tumour recurrence. ${ }^{12}$ The patient presented in this article was treated with a complete resection of the tumour. The application of imatinib mesylate for the GIST treatment was a successful introduction of molecularly targeted therapy for the treatment of solid tumours. The imatinib therapy is used for the treatment of unresectable cases, recurrent or metastatic cases. Surgery or ablative modalities can be used when the disease becomes amenable to gross resection due to changes initiated by imatinib, or when the tumour develops resistance to imatinibe treatment. 8,12 The evaluation of the GIST treatment with imatinib mesylate is usually performed with $\mathrm{CT}$, sometimes with ${ }^{18}$ FDG-PET. The problem in the evaluation of the GIST treatment with imatinib mesylate is that they correlate poorly with currently internationally agreed classifications for the evaluation of response to the treatment of solid tumours like the World Health Organization criteria or the Response Evaluation Criteria in Solid Tumors (RECIST) criteria. It is suggested that changes in tumour density on CT scans are better modes of assessment of the response to the imatinib treatment than an internationally agreed guidelines. The minority of patients with GIST, less than $15 \%$, have a primary resistance to imatinib mesylate. Half of the patients will develop resistance 2 years after the initiation of the 
imatinib treatment, usually because of the secondary KIT mutation. ${ }^{12}$ Generally, the median time of recurrence of GISTs after the surgery is 19 to 25 months with the 5year survival rate about $50 \%{ }^{11}$

GIST may be statistically rare mesenchymal tumour of the gastrointestinal tract, but whenever the large heterogeneous, abdominal mass with higher attenuation on periphery and lower in the central parts is depicted on the contrast-enhanced CT scans, GIST must be firstly put on the differential diagnosis list. That is important because the transabdominal biopsy of the tumour is not recommended because of the possibility of seeding the tumour cells. ${ }^{16}$

\section{References}

1. Miettinen M, Lasota J. Gastrontestinal stromal tumors: review on morphology, molecular pathology and differential diagnosis. Arch Pathol Lab Med 2006; 130: 1466-78.

2. Levy AD, Remotti HE, Thompson WM, Sobin LH, Miettinen M. Fronm the Archives of the AFIP Gastrointestinal stromal tumors: radiologic features with pathologic correlation. Radiographics 2003; 23: 283-304.

3. Kachanov DY, Dobrenkov KV, Shamanskaya TV, Abdullaev RT, Inushkina EV, Savkova RF, et al. Solid tumors in young children in moscow Region of Russian Federation. Radiol Oncol 2008; 42: 39-44.

4. Miettinen M, Lasota J. Gastrointestinal stromal tumors - definition, clinical, histological, immunohistochemical, and molecular genetic features and differential diagnosis. Virchows Arch 2001; 438: 1-12.

5. Suster S. Gastrointestinal stromal tumors. Semin Diagn Pathol 1996; 13: 297-313.

6. Levy AD, Remotti HE, Thompson WM, Sobin LH, Miettien M. Gastrointestinal stromal tumors: radiologic features with pathologic correlation. Radiographics 2003; 23: 283-304.
7. Kindblom LG, Remotti HE, Aldenborg F, MeisKindblom JM. Gastrointestinal pacemaker cell tumor (GIPACT): gastrointestinal stromal tumors show phenotypic characteristics of the interstitial cells of Cajal. Am J Pathol 1998; 152: 1259-69.

8. Blay J-Y, Bonvalot S, Casali P, Choi H, DebiecRichter M, Dei Tos AP, et al. Consensus meeting for the management of gastrointestinal stromal tumors. Report of the GIST Consensus Conference of 20-21 March 2004, under the auspices of ESMO. Ann Oncol 2005; 16: 566-78.

9. Miettinen M, Sobin LH, Lasota J. Gastrointestinal stromal tumors of the stomach:a clinicopathologic, immunohistochemical, and molecular genetic studies of 1765 cases with long-term follow-up. Am J Surg Pathol 2005; 29: 52-68.

10. Dematteo RP, Lewis JJ, Leung D, Mudan SS, Woodruf JM, Brennan MF. Two hundred gastrointestinal tumors: recurrence patterns and prognostic factors for survival. Ann Surg 200; 231: 51-8.

11. Emory TS, Sobin LH, Lukes L, Lee DH, O'Leary TJ. Prognosis of gastrointestinal smooth-muscle (stromal) tumors: dependence on anatomical site. Am J Surg Pathol 1999; 23: 82-87.

12. Gold JS, DeMatteo RP. Combined surgical and molecular therapy: the gastrointestinal stromal tumor model. Ann Surg 2006; 244: 176-84.

13. Ludwig DJ, Traverso LW. Gut stromal tumors and their clinical behavior. Am J Surg 1997; 173: 390-4.

14. Sharp RM, Ansel HJ, Keel SB. Best cases from the AFIP: gastrointestinal stromal tumor. Armed Forces Institute of Pathology. Radiographics 2001; 21: $1557-60$.

15. Pidhorecky I, Cheney RT, Kraybill WG, Gibbs JF. Gastrointestinal stromal tumors: current diagnosis, biologic behavior, and management. Ann Surg Oncol 2001; 8:50-9.

16. Burkill GJ, Badran M, Al-Muderis O, Meirion Thomas J, Judson IR, Fisher C, et al. Malignant gastrointestinal stromal tumor: distribution, imaging features, and pattern of metastatic spread. Radiology 2003; 226: 527-32.

17. Phongkitkarun S, Phaisanphrukkun C, Jatchavala J, Sirachainan E. Assesment of gastrointestinal stromal tumors with computed tomography following treatment with imatinib mesylate, World J Gastroenterol 2008; 14: 892-8. 
18. Hong X, Choi H, Loyer EM, Benjamin RS, Trent JC, Charnsangavej C. Gastrointestinal stromal tumors: role of $\mathrm{CT}$ in diagnosis and response evaluation and surveillance after treatment with imatinib. Radiographics 2006; 26: 481-95.

19. Hama Y, Okizuka H, Odajima K, Hayakawa M, Kusano S. Gastrointestinal stromal tumor of the rectum. Eur Radiol 2001; 11: 216-9.

20. Ghanem N, Altehoefer C, Furtwängler A, Winterer J, Schäfer O, Springer $\mathrm{O}$, et al. Computed tomography in gastrointestinal stromal tumors. Eur Radiol 2003; 13: 1669-78.

21. Miettinen M, Sarlomo-Rikala M, Sobin LH, Lasota J. Gastrointestinal stromal tumors and leiomyosarcomas in the colon: a clinicopathologic, immunohistochemical, and molecular genetic study of 44 cases. Am J Surg Pathol 2000; 24: 1339-52.

22. Miettinen M, Lasota J. KIT(CD117): a review on expression in normal and neoplastic tissues, and mutations and their clinicopathologic correlation. Appl Immunohistochem Mol Morph 2005; 13: 205-20.

23. Sarlomo-Rikala M, Tsujimura T, Lendahl U, Miettinen M. Patterns of nestin and other intermediate filament expression distinguish between gastrointestinal stromal tumors, leiomioma and scwannomas. APMIS 2002; 110: 499-507. 